\title{
Moball Network: A Self-Powered Intelligent Network of Controllable Spherical Mobile Sensors to Explore Solar Planets and Moons
}

\author{
Faranak Davoodi ${ }^{1}$ \\ Jet Propulsion Laboratory, Pasadena, California, 91109, USA \\ Joel Burdick ${ }^{2}$ \\ California Institute of Technology, Pasadena, California, 91125, USA \\ and \\ Mina Rais-Zadeh ${ }^{3}$ \\ University of Michigan, Ann Arbor, Michigan, 48109, USA
}

\begin{abstract}
This paper introduces a novel planetary exploration system capable of functioning in most solar planets and moons. The Moball Network is an agile, self-powered, intelligent, and reconfigurable distributed in-situ exploratory system. We compare our proposed system with state of the art planetary exploration systems and concepts and suggest several novel mission architectures specifically for planetary bodies with an atmosphere such as Mars, or those whose surface includes lakes and ice sheets such as Titan or the Earth's northern and southern caps, as well as smaller bodies with no atmosphere such as the Moon or asteroids. We also describe the current development status of the system and the proof of concept for the ideas presented.
\end{abstract}

\section{Introduction}

$\mathrm{A}_{\mathrm{o}}$ ddressing the increasing importance having a cost-effective and game changing exploratory system, we introduce a novel multi-planetary exploration system and architecture for studying the surface of planetary bodies such as the Moon, Mars, Titan, or asteroids, using an intelligent network of controllable spherical mobile sensors called Moballs $^{1-4}$. A Moball Network is envisioned to be a scalable, self-powered, controllable, distributed system that would share tasks intelligently in order to optimize its resources (power, memory, and bandwidth) and the performance of its exploring and sensing responsibilities. The low-mass self-rigidizable Moball design allows for a large number of units to be packed in an aeroshell, resulting in a Moball Network which can in-situ map a wide range of phenomena across a much larger expanse of the surface compared to many other proposed systems. It could substantially improve the possibility of finding rare

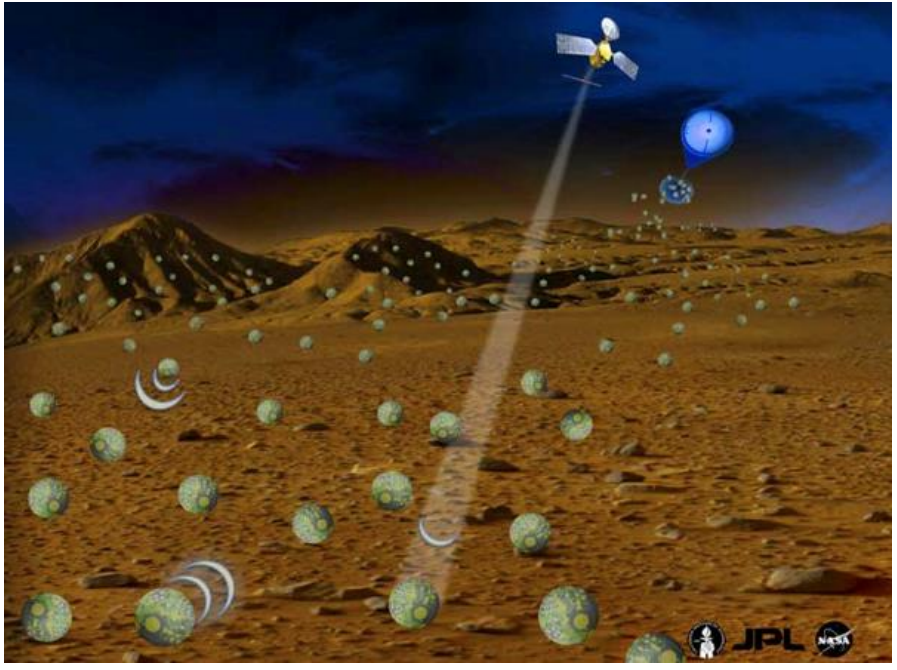

Figure 1. A wireless mesh network of Moballs use low risk deployers such as parachutes to get distributed over a large expanse of the surface to explore Mars

\footnotetext{
${ }^{1}$ Technologist, Engineering and Science Directorate, 4800 Oak Grove Dr./ T1708-104, Pasadena, CA 91109

${ }^{2}$ Professor, Mechanical and Civil Engineering, 1200 E. California Boulevard/ MC 155-44, Pasadena, CA 91125

${ }^{3}$ Assistant Professor, Electrical Engineering and Computer Science, 2406 EECS, Ann Arbor, MI 48109
}

American Institute of Aeronautics and Astronautics 
phenomena such as bio signatures and the valuable samples to be returned to Earth, through a random dynamic search. The Moball-buoy Network is a resilient and fault tolerant exploratory system; even if many of the sensors go down, the other units of the system can still work and help the mission go on.

It could explore difficult terrains that were beyond the reach of previous missions, such as regions with very steep slopes, cluttered surfaces and/or sand dunes; Moball Network is envisioned to be an ongoing mission with a long life span. It could achieve many of NASA's scientific objectives in a cost-effective way, leaving a long lasting sensing and searching infrastructure on the planetary surface.

\section{System Summary of Moball Network}

The Moballs can use wind, gravity, or their self-powered internal propulsion mechanism to move. The Moballs have peer-to-peer communication with each other. They also have communication capabilities with the orbiters and other in-situ assets, when in view. This comprises a wireless mesh network shown in Fig. 1, which provides an interface for a global controller to create a harmonious distributed exploratory system.

The Moballs are spherical, light, and bouncy; they weigh 2500 - $4500 \mathrm{~g}$ (depending upon their energy harvesting and science payload configurations). They could be made of impact and tensile resistance light elastomers such as Ethylene Tetra Fluoro Ethylene (ETFE), Polyurea, or Kevlar; their elastic tightening frames are made of material such as fiberglass or Titanium; they have low-mass low-power, or imprinted, sensors and electronics. This will make the Moballs light and bouncy while sturdy enough to withstand the impact of their initial fall, as well as other impacts from obstacles in their way. It also makes them agile and easy to move in various directions or pass through cluttered terrains, sand dunes, or steep slopes.

Moballs are wind-opportunistic; when the Moballs are deployed in planetary bodies with an atmosphere such as Mars, their being bouncy and lightweight, allows them to take advantage of wind for their mobility and energy harvesting.

Moballs are self-rigidized structures. Many deflated Moballs can be pressurized in aeroshell containers before deployment. During deployment, the Moballs would be inflated using self-rigidizing techniques to thereafter hold their inflated spherical structure. The Moball's rigidized
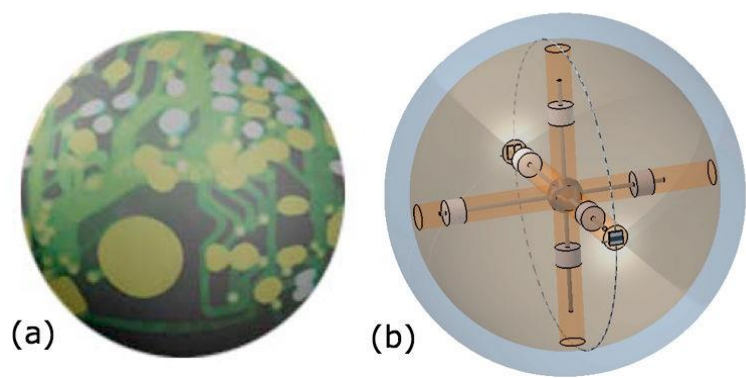

Figure 2: (a) The Moball is a bouncy, selfrigidizable spherical multifunctioning sensor with elastic shell and the laminated imprinted electronic circuits, antenna, solar cells, imagers, etc. (b) The Moball's dual functioning Mechanical Control and Linear Induction System ${ }^{1-4}$ consists of three internal perpendicular axes with movable and controllable magnetic weights moving within solenoids. The controlled movements of the magnetic weights (via the solenoids) along each of the three axes can induce rolling motions to enable precise positioning during science investigations. During wind or gravity driven movement, the controlled mass movements can bias the Moball's motion. Alternatively, the uncontrolled movements of the masses can generate electric power as they move inside the solenoids. The Moball Network can also be used on windless bodies (e.g. Moon, Europa, asteroids) exploiting solar panels or thermoelectricity techniques. structure will eliminate the use of air pumps (and the resulting power and system complexity needed to keep the sphere inflated over its entire exploration mission).

The Moball Network is scalable; the software in the Moballs and satellites can get upgrades. Furthermore, new Moballs and satellites with new functionalities could be added.

The Moball Network is cost-effective; the Moballs use natural resources to harvest the energy they need and do not need hefty and expensive propulsion and mobility equipment. Moreover, the Moball Network does not need a sophisticated, heavy and costly lander. Since they are much lighter, the rocket payload can consist of hundreds of Moballs. For comparison, the weight of the Mars Science Lab was around $900 \mathrm{~kg}$. With Moballs weighing $3 \mathrm{~kg}$ each, say, one could send 300 Moballs with the same payload to Mars 
The Moball is controllable; they use their internal mechanics, as shown in Fig. 2, to initiate moving, to stop, or to control their speed and trajectory, in the presence or absence of wind. The dual functioning Moball's mechanical control and energy induction system consists of magnets moving within solenoidal tubes aligned along three internal axes. Controlled movements of the magnets (via the solenoids) can induce rolling and steering motions to enable precise positioning and tracking. The controlled magnet-mass movements can bias the Moball's motion in the presence of the wind, when it exists, or initiate rolling in the absence of wind.

Moballs could detect and process hazardous objects, such as gullies, large obstacles, or hazardous events such as dust devils, and plan to avoid them. As in Fig. 3(a) and Fig. 3(b), the Moball can change its center of mass in order to hop over the obstacles. The initial speed of the Moball, caused by wind, gravity, or its own internal propulsion system, will make the hopping of the Moballs over an object be more likely, compared to the state of art with the planetary hoppers (e.g. hedgehogs) where the vehicle hops from a still state. The Moball can stop by coordinating the motions of the solenoid magnets and lowering its center of mass inside the sphere. As in Fig. 3(c), the Moball can steer away from the obstacles by adjusting its center of mass to lean on one side.

The Moballs are self-powered; they use natural resources on the planetary bodies that they are deployed to harvest the energy they need for their sensing, mechanics, data processing and communication. Moballs can exploit sunlight, wind, gravity driven motion and vibrations, or diurnal temperature differences using thermo-electricity techniques; to continually harvest the energy needed for mobility control, sensing, and communications.

The uncontrolled magnet oscillations of the Moball's dual functioning mechanical and energy harvesting system shown in Fig. 2, caused by wind or down rolling movement of the Moballs, inside the tubes covered by solenoids can generate electric power. The Moball's capability of energy harvesting exploiting wind or gravity-driven motions or using thermoelectricity techniques, will allow the Moballs to harvest energy even when it is dark (e.g. during winter in the northern or southern caps of the Earth, Mars, or Titan), or when the sunlight is very limited (e.g. on Mars when it is dusty, or
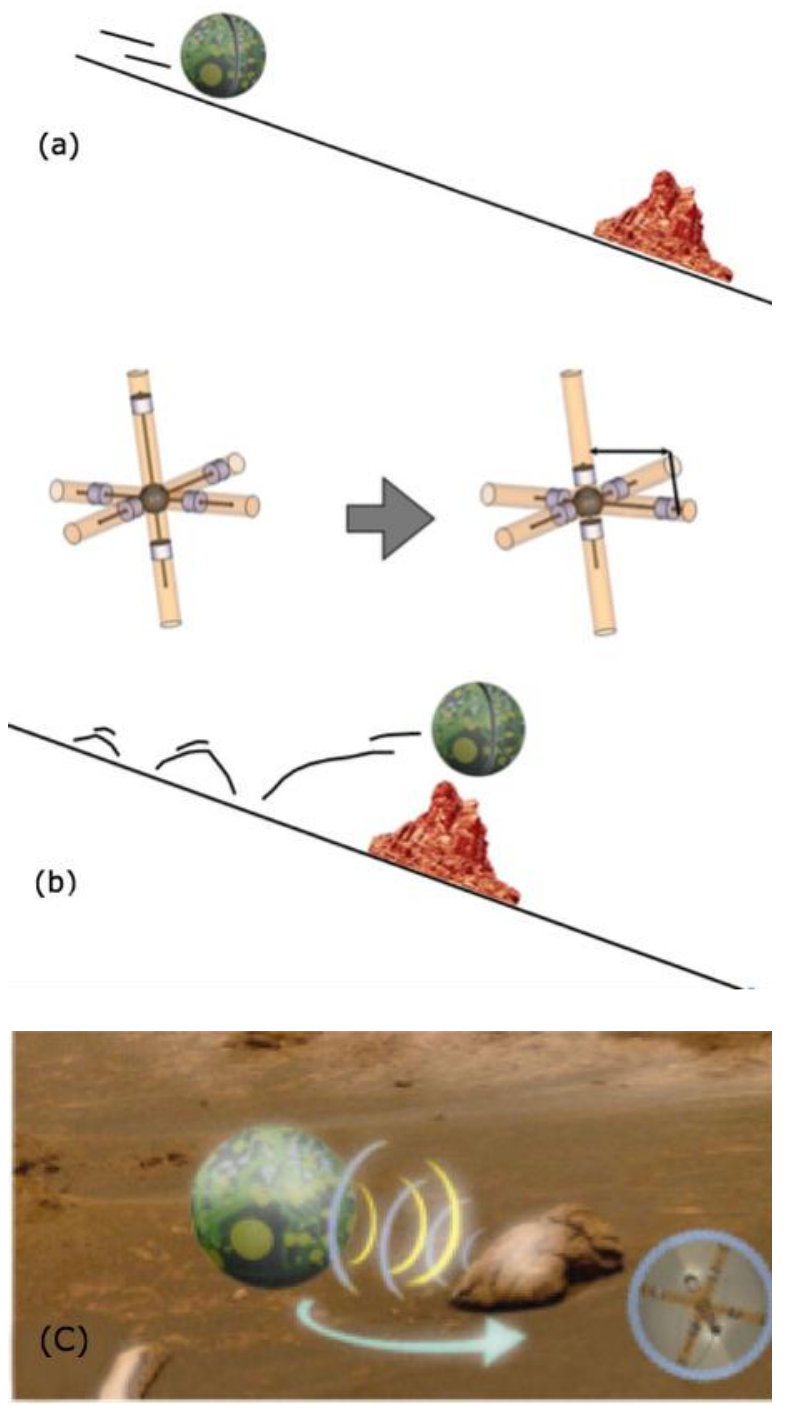

Figure 3: (a) The Moball detects and process obstacles and plans to avoid them (b) The Moball can change its center of mass in order to hop over obstacles. The initial speed of the Moball makes its hopping more likely.

(c) Moball can coordinate its magnet-weights to stop or to steer away from the obstacles. 


\section{Mission Scenarios Using the Moball Network}

The Moballs could be released using a deployer sent to the planetary bodies. This would be done at a correct altitude and with an appropriate velocity to ensure widespread and safe landing. Various strategies for deployment could be envisioned, such as sending multiple aeroshells and parachutes to several different geographic locations of Titan or Mars, which would release the Moballs over a vast area of interest--think of pollination.

Conversely, as in Fig. 4, a low risk deployer such as a parachute could drop several resilient and elastic Moballs on top of a planetary mountain. The Moballs could use gravity to traverse and explore the mountain all the way down. Planetary mountains could be great choices for future exploration missions. For example, scientists suggest that Arsia Mons might be a valuable exploration site for possible biosignatures due to its glacio-volcanic environment 200 million years $\mathrm{ago}^{12}$. The current state of art for explorations, including the heavy and slow rovers with high-risk landers, might not be appropriate for such exploration sites.

Two major classes of Mission architecture for the Moball Network are described below:

\section{A. Multiple Aeroshell Moball Network Missions}

We suggest a novel Multiple-Aeroshell Moball Network Mission class of mission architectures, which we believe would decrease the cost, time, and risk associated with future exploratory missions. For

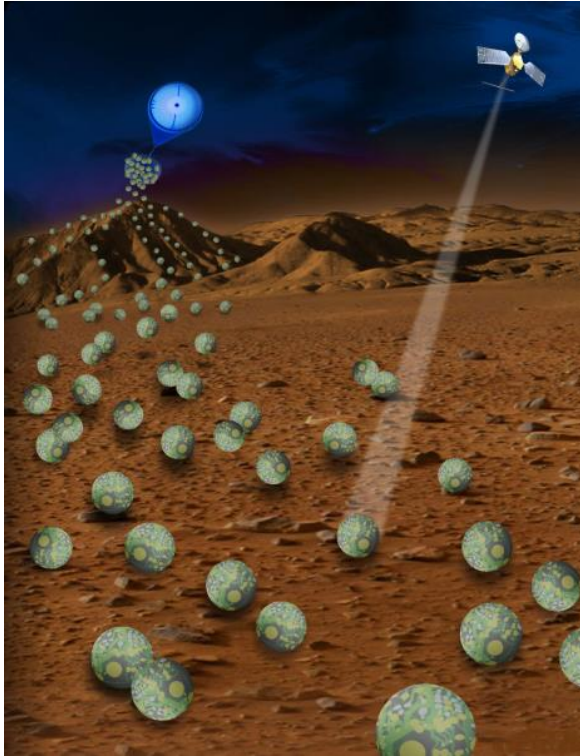

Figure 4: A parachute could drop Moballs on top of the planetary mountains to explore it all the way down. example, a single spacecraft could place two aero-shells in the orbit of Mars (or other planetary bodies). The Moball-aeroshell carries the compressed Moballs while the other rover-aeroshell carries a rover or lander. The rover-aeroshell would remain in orbit while the Moball-aeroshell descends to the area of interest (using steering thrusters similar to MSL's aeroshell, or perhaps a large parachute) to distribute Moballs over a large surface area. The self-rigidizable Moballs will be inflated just after the deployment.

Depending on the environment and the altitude at which the Moballs are released, smaller parachutes attached to each Moball can enable safe landing (the chutes can be jettisoned after landing). The quickly spreading Moballs would use various on-board imagers, spectrometers, and sensors to identify and map the relational location of the most important and valuable sampling targets and environmental phenomena which would need more sophisticated examination by rovers. Based on the map prepared by the Moballs, the rover's descent could then be targeted to the region covering as many important identified samples as possible. Considering the very slow speed of conventional rovers (MSL's travels $\sim 30$ meter/hour), this approach will save time, cost, and risk for the mission.

Furthermore, the Moballs could provide real time information and guidance directly from the landing site to the rover's descent control system regarding the environmental situation (e.g. wind speed, dust conditions, obstacles in the area) and the descending aeroshell's landing parameters (e.g. feedback on its speed, angle, direction) in order to help it land safely and precisely at the chosen landing spot. The Moballs can also potentially act as visual landmarks or radio beacons to improve the accuracy of the descent control. Another example of a Multiple-Aeroshell Moball Network Mission will be to place multiple smaller aeroshells containing Moballs into a planetary orbit, and then disperse Moballs to different geographical locations (e.g., one to the southern and the other to the northern hemisphere). The Moballs could be customized for each specific environment. The Moball network design can cover larger surface areas at a significantly reduced cost. The larger coverage will allow scientists to make more informed inferences and to understand geophysical trends. It will also allow them to identify "rare" events such as Martian "blueberries." Cooperating Moballs in close proximity can generate 3-dimensional reconstructions of a geographical area.

Since Moballs could be used as scouts for planetary rovers, and could detect the closest feasible landing location near important science targets, they can save time and cost for future missions. And since they could also be used as beacons, it could make the landing of the rovers and in general future missions less risky, and therefore more readily funded. 


\section{B. Phoenix-like landers and the Moball Network}

Another novel mission architecture that we suggest for the Moball Network is sending Phoenix-like landers containing compact Moballs integrated inside the lander's structure to a planetary body to explore. This is especially useful for those planetary bodies that we want to visit for the first time, such as Titan, Europa, or the asteroids. This way the lander's more sophisticated instruments, drills, and robotic tools can perform detailed tests on the environment, surface and under the surface of the planetary body, while more agile Moballs could go around and explore the large expanse of the planetary body and gather pictures and overall information about the newly visited planetary body in a much faster pace. The Moball Network's being self-powered and self-contained will allow constant exploration of the surface of the planetary body, looking for the most valuable areas with important samples and also determining the most appropriate landing sites close to them in the future missions

Depending on the size and environment of the planetary body that the exploratory Moballs are sent to, we might have different deploying strategies for sending the Phoenix-like lander and Moballs. For example, for larger planets with an atmosphere (e.g. Titan or Mars), the lander could release the Moballs from a higher altitude, in order to spread them in a larger expanse before touching down on the surface. The larger surface of the Moballs (spheres with 1-2 meter in diameter) and the body's atmosphere will protect the Moballs from the initial impact. However, for bodies with no atmosphere, such as the Moon, the lander could first touchdown on the surface and then release its Moballs, which were pressurized in a container inside the lander's structure. For very small bodies such as asteroids, there will be a problem when the Moballs are deployed from a higher altitude, since the Moballs could bounce back from the surface and go out of control and reach. Therefore, in order to deploy the Moballs, the Phoenix-like lander will first land and let a ramp that was integrated in the structure be ejected out from the container holding the Moballs and down to the surface. The Moballs can then roll down slowly to the surface using their internal mechanics shown in Fig. 2.

\section{The Distributed Control Architecture for the Moball Network}

Our proposed system is much more than a collection of sensors: the system is larger than the sum of its parts. In addition to communicating with orbiters, the Moballs can communicate with each other and store data and commands for delayed relaying and execution. The system therefore constantly updates a global map of where the Moballs are, where they are heading, and what each Moball's energy and memory resources are. As a result, the Moballs can distribute tasks, data, computations, etc., and cooperate in intelligent ways. For example, if several Moballs sense that they are in close proximity to one another they need not all perform the same task; one Moball could measure the local temperature, the other the local vapor content, etc., thereby saving the system power and reducing data traffic. On the other hand, if for some reason, highly reliable data is required, the Moballs can fuse

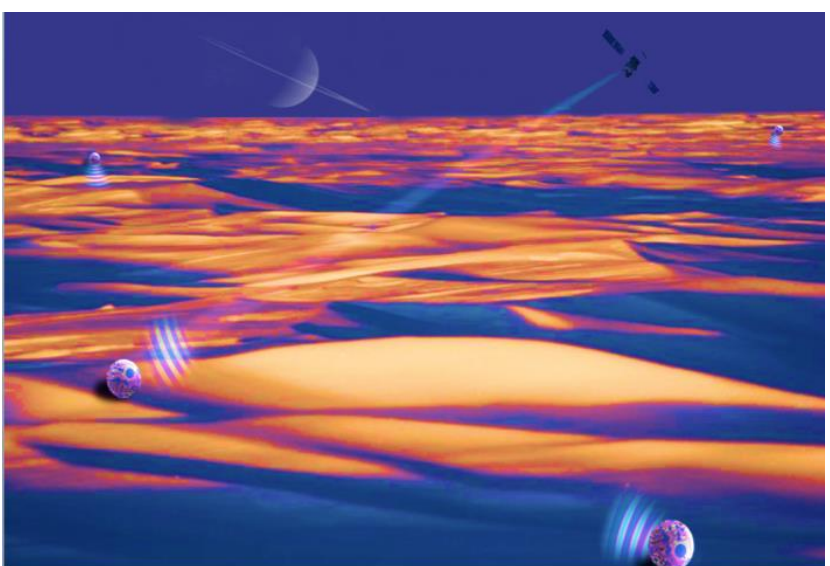

Figure 5: The Moballs share tasks and learn from each other. They can also fuse their data and send reliable and concise data back to the orbiter and Earth. their measurements (by averaging, say) and send much more accurate values to the satellite and Earth.

As in Fig. 5, the Moball Network configured as a wireless mesh network can support a global communication and control system that will ensure desired area coverage, avoidance of hazardous objects, events or areas, and the optimization of the Moballs' explorations of areas of high scientific value (according to their available resources-power, memory, bandwidth).

Moballs have a stop-and-go capability. They can be "anchored" in moderate winds by lowering their center of mass; conversely, when necessary, or when conditions are suitable, their center of mass can be moved upwards to resume their wind-drive motion. As a result, the motion and distribution of a group of Moballs can be controlled either through a local planning, through negotiation among neighboring Moballs, or through a centralized command (e.g., by scientists and technologists on Earth). In this way Moball Network could dynamically reconfigure itself using an opportunistic wind-propelled motion strategy. 
We envision a Moball Network as a system which can learn from previous experience or other Moballs in order to identify hazardous areas safe traversal paths, or significant objects.

Using ranging information provided by the peer-to-peer communications, or from an orbiting satellite, as well as fused IMU data the Moball positions can be estimated, thereby allowing them to adjust their distances from each other in order to maintain networking and to collectively explore an area as a united body.

The Moballs can also move in the absence of wind. They can exploit the mechanical control system shown and explained in Fig. 2 and use their internal propulsion system (e.g. a rechargeable battery, charged by various suggested energy harvesting techniques) in order to initiate movement to an area or object of interest (for example, an interesting rock that needs more examination, or from shade to sun in order to harvest more energy) or to avoid hazardous objects or areas. .

The Moball Network's distributed control system might ensure that Moballs intelligently share mission tasks that have been defined for different areas in order to optimize performance with respect to overall mission exploration goals as well as the usage of the system resources (power, memory, and the bandwidth). For example, when several Moballs are in close proximity, not all of them need perform the same tasks. Instead, one might measure temperature and pressure, another one might take pictures, and another might stop to perform spectrometry tests on a certain rock. The acquired data might then be transferred to a different Moball, which will be tasked with sending the data to an orbiter (perhaps because that Moball has better line-of-sight communication with the orbiter). In this fashion, system power and memory can be used intelligently respecting bandwidth limitations. The Moballs in an area can also fuse their information (say by averaging) in order to report more accurate data back; fused data is also more compressed, which will decrease communication traffic.

Predefined preferences on possible events can be prioritized. For example, recognizing particular types of rocks, bio-signatures, or chemical compositions, or monitoring a passing dust devil may have a higher ranking and therefore require more attention and tests. Not only would ranking potentially save energy and system bandwidth, when a Moball encounters a highly ranked event, it can ask for help from neighboring Moballs to assist the "important" Moball in collecting, processing, and communicating its data. As a result, the "important" Moball can focus its resources on sensing, measuring and recording high priority events and not be worried about lack of memory. The ranking algorithm also comes in handy when a Moball needs to decide to get rid of its older, lower ranking, data when it is short on memory. Furthermore, when it is short on power and an orbiter happens to be passing by, it is worth only to transmit the higher-ranked events.

The orbiting system is a major component of the Moball Network architecture. It facilitates the communication of the Moballs with each other and with Earth, it performs power- and memory-intensive computational tasks, it gives optimized task plans to Moballs in a centralized fashion, and it updates and broadcasts the Moballs' topology map and the level of their memory and energy. Since the orbiters know the current location of the Moballs and are likely to have access to wind circulation patterns, they can "predict" the near future locations of the Moballs. The orbiters can therefore inform the Moballs of their proximity to high-ranking events so that they can conserve their memory and power in anticipation of an important encounter. The knowledge of Moball locations by the orbiter can also be used to organize and prioritize inter-Moball communication

\section{Comparison to other approaches \& concepts}

Compared to recently proposed mission concepts that share some similarities to what is proposed here, a Moball Network has many advantages.

1) Unlike the Tumbleweed ${ }^{5}$ rovers which act as single wind-driven rovers, and Microbots ${ }^{8}$, hedgehogs ${ }^{9}$, which are not wind-driven and need a lot of energy for their locomotion (using their mechanics to hop around), the Moball Network can take advantage of wind for its mobility and is a distributed system that share tasks intelligently in order to optimize its resources (power, memory, bandwidth) and performance of its exploration, sensing, and positioning responsibilities across a whole network of lightweight sensor platforms. Also, using the control mechanics in Fig. 2, Moballs can move even in the absence of wind.

2) The Tumbleweed is "assumed to be a perfect, inelastic, homogeneous sphere that rolls without slip"5; hedgehogs also "move nimbly and precisely" 8 . In contrast, the elastic and bouncy Moballs can be extremely agile, rebounding from impact with an obstacle, and can saltate by the turbulent movements of the wind to easily pass through cluttered surfaces without requiring a lot of energy; especially, given that the Moball's preferred height for Mars deployment is about 1.5-2.0 meters, and most boulders on Mars are less than 1 meter tall ${ }^{10}$.

3) The Mechanical Control and Linear Induction System suggested for the Moballs described in Fig. 2 is novel. This compact, robust, and lightweight design enables both crucial energy harvesting needed for long duration missions, and precise positioning control authority needed for science. 
4) While the use of a pendulum for energy harvesting has been suggested for the tumbleweed design $^{6}$, this approach is not suitable for tumbling Moballs, as the internal space occupied by the pendulum is excessive, energy is harvested only for specific rolling motions, and the required mass is high. The magnetic weights in the Moball's structure move linearly and securely in their solenoidal tubes covered. Any rolling or bouncing motion can induce electricity.

5) No energy harvesting has been suggested for the mechanics of the Microbots and hedgehogs.

6) Microbots ${ }^{8}$ and hedgehogs ${ }^{9}$ need a great deal of energy for their motility and therefore their activity is very limited if used on cluttered and windy Mars (or Titan). The Microbots' life span is just 30 days. Also, dust on Mars and fog on Titan limits the solar energy that could be harvested by hedgehogs.

7) The mission strategies for the Moball Network suggested in the section III, the multiple-aeroshell deployment, and the combination of Phoenix-like landers and the Moballs, are novel. The use of low cost and parallel "scouts" to improve a rover's landing site value is also novel.

8) The autonomous and distributed control architecture suggested for the Moball Network, described in IV, is novel. The hedgehogs are not autonomous and Microbots don't reconfigure themselves based on the priority of the region and the resources available.

\section{Technical Details and Rationale}

Prototypes of the main Moball sub-systems (structural shell, mechanical energy harvesting and control system, on-board computing and sensor processing, and energy management) are currently in development. This section summarizes some of the preliminary calculations and simulations, which support the feasibility of future Moball devices

\section{A. Mass and Volume Budget}

The Moball mass will determine the amount and type of sensors that can be incorporated into its structure, while its volume will influence the amount of energy that can be harvested (since the energy scavenged from thermoelectricity, piezoelectric, the wind-driven motion, etc., depends on the volume available). The Moball mass and volume cannot be chosen arbitrarily. They are tied together by virtue of the fact that they should allow for winddriven motion under the typical conditions. Considering surface conditions on Mars (wind speed of 10-15 m/s, atmospheric density of $20 \mathrm{~g} / \mathrm{m}^{3}$ ) and Titan (wind speed of $1 \mathrm{~m} / \mathrm{s}$, atmospheric density of $1.88 \mathrm{~g} / \mathrm{cm}^{3}$ ), using formulas developed in previous studies ${ }^{4}$ a diameter of $\sim 1.5$ meter is sufficient to guarantee a wind-driven motion for a Moball with mass of 500-5000 grams.

\section{B. Energy Budget and suggested sensors}

A key issue for energy-scavenging systems is the level of available power, and whether this power is sufficient for operation. At any instant, the power which can be scavenged is a function of the conditions of the Moball's environment, which are apt to vary significantly. In Fig. 6 , a summary of power vs. volume for some of the most recent power scavengers (primarily inertial and thermal) is depicted, highlighting the different transduction technologies, including piezoelectric (PE), electromagnetic (EM), electrostatic (ES), and thermoelectric (TE). The most important conclusion is that most scavengers produce on the order of 10$100 \mu \mathrm{w} / \mathrm{cm} 3$ for smaller devices, and perhaps on the order of several $100 \mu \mathrm{W}$ to several $\mathrm{mW}$ per $\mathrm{cm} 3$ for larger systems.

We have performed a more detailed study of the solenoidal mechanical energy harvesting and control system ${ }^{4}$. A

$2 \mathrm{~m}$ diameter Moball can potentially generate at least $\sim 2$ watts continuously during wind-driven motion in the Earth's polar regions. Scaling this result to Mars yields an

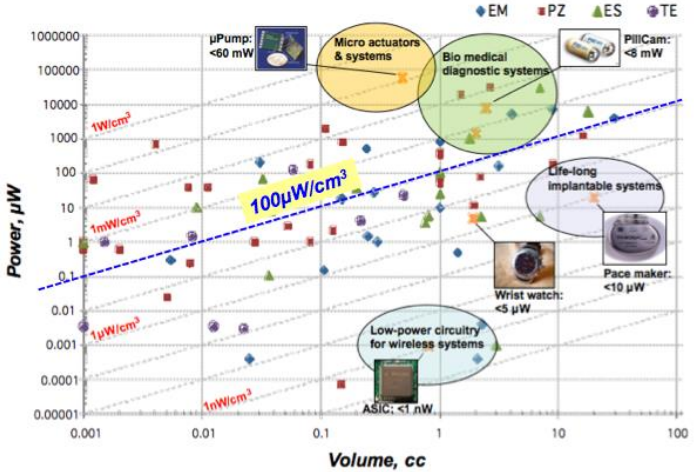

Figure 6. Power generated Vs. Volume for most recent power scavengers ${ }^{7}$ expected $500 \mathrm{~mW}$ of continuous energy generation during typical wind-driven motions. Assuming scavenging for 8 hours per Martian sol, one can expect to harvest 3500-4500 Joules of energy per sol. This amount of energy can operate many sensors, an onboard microprocessor, and communication system. Moball sensors and micro-devices 
that fit within this energy budget could include a MEMS Inertial Measurement Unit (IMU consisting of 3-axis gyro, accelerometer, and magnetometer), CMOS Panoramic Imager, Silicon MEMS Pressure Sensor, TES bolometers, MEMS wind vector sensors, uncooled infrared cameras, acoustic sensors (for Titan), Raman spectrometer with a VLOK microchip laser integrated within a Raman probe-head, Multispectral Microscopic Imager, Miniature Mass Spectrometer (MMS), Compact curved focal plane array camera (UV/Vis/NIR) with a large field of view, MEMs Gas detectors, microfluidics, and bioMEMS chips.

\section{RF Communications Link Budget}

Although the level of communication required by the heads up and collaborative task sharing strategies of the Moball may look power-intensive, the Moballs are programmed to keep expensive satellite communications to a minimum, and use low power micro-RF for peer-to-peer communication. Assigning a $100 \mathrm{~mW}$ continuous transmit power, more than 10 hours of consecutive peer-to-peer communication per sol is possible while still allowing for onboard sensing and computation. In order to increase the communication distance with reasonable data rate, QPSK modulation can be used for both uplink and downlink communication. QPSK provides a spectral efficiency of $\sim 1.6$ bits/Hz and a required SNR of $\sim 14 \mathrm{~dB}$ for $10^{-6}$ bit-error-rate (BER). The frequency band of $433 \mathrm{MHz}-434 \mathrm{MHz}$ available for ISM applications is chosen due to longer propagation distance with appropriate antenna size $(<10 \mathrm{~cm})$, which is less than the diameter of the Moball. Based on this assumption, the link budget the realizable communication distance is estimated to be $100 \mathrm{~km}$ between Moballs with the maximum date rate of $320 \mathrm{~kb} / \mathrm{s}$.

\section{Current Status}

Our initial prototyping efforts have focused on optimizing and validating the mechanical self-energy harvesting and motion control system ${ }^{4}$. Through extensive Finite Element Modeling we have studied the maximum energy that can be generated from the solenoidal tubes of Fig. 2 using a moving mass system having the geometry shown in Fig. 7 (a), consisting of a central Neodymium Iron Boron magnet and low carbon "back-iron" end caps. This geometry shapes the magnetic field so that the majority of the field lines in the combined system are normal to the solenoid axis, thereby maximizing the harvested energy for a minimum combined mover mass. Fig. 7 (a) shows a prototype of a single axis (out of 6 total axes) of the energy harvesting system, consisting of moving magnet, solenoidal coil, and magnet guides. Fig. 7 (b) shows the voltage generated as the magnet is dropped through the coil while the magnet's motion is parallel to gravity. Note that the voltage produced by the coil, as well as the power produced by the magnet motion, is a function of the load's electrical impedance. For the design presented in Fig. 7 (a) and Fig. 7 (b), we found that peak energy produced by one pass of the magnet through a coil was 2.3 joules. When scaled to a 2-meter diameter Moball operating in typical wind Arctic wind conditions of 4-6 m/sec, this result shows that such a device can generate 3-5 watts of continuous power.

This amount of power is sufficient to: (1) operate a low power ARM cortex dual-core processor; (2) power a suite of on-board sensors; (3) drive a $100 \mathrm{Kbit} / \mathrm{sec}$ communication system (with inter-Moball separation of up to $12 \mathrm{~km}$ ); and (4) charge an on-board battery to drive the motion control system during windless conditions.

Moreover, we have shown that on a flat terrain in the Arctic, the
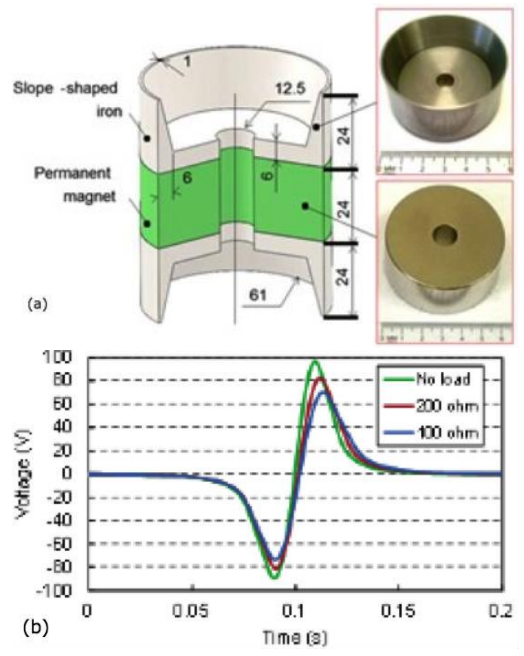

Figure 7: (a) Design of the Moball's moving magnet. (b) Voltage vs. time while moving magnet passes through the solenoidal coil center, under different circuit loading

\section{conditions}

Moball needs only 2 watts to make low speed rolling motions (about 1 meter/sec), assuming there is no wind to help the motion. On the same terrain, we need only 1-to $1.5 \mathrm{~m} / \mathrm{sec}$ wind to start the mobile rolling (and therefore using no on-board power to drive the motion). For the 2-meter diameter Moball that we sized for the Arctic, at a wind speed of 5-6 meters/second, the Moball will roll at 2.5-3.0 meters/sec according to our simple models.

\section{Conclusion}

While the Moball design is seemingly simple, a fleet of networked Moballs can be deployed in a cost-effective way to provide wide-area, persistent and sophisticated coverage of planetary surfaces. Their networking capabilities also provide high science throughput with high resiliency and redundancy. We believe that this architecture offers many potential advantages for future space exploration initiatives. 


\section{Acknowledgments}

The research was carried out at the Jet Propulsion Laboratory, California Institute of Technology, under a contract with the National Aeronautics and Space Administration (NASA).

\section{References}

${ }^{1}$ Davoodi, F., Hajimiri, A., Murphy N., Mischna, M., Nesnas, I., Nikzad, S., Nesmith, B. "Gone with the Wind on Mars (GOWON): A Wind-Driven Networked System of Mobile Sensors on Mars" Concepts and Approaches for Mars Exploration, LPI 2012

${ }^{2}$ Davoodi, F., Davoudi, F. "Design for the structure and the Mechanics of Moballs". NASA Technical Briefs, October 2012. 36(10):p.66-68.

${ }^{3}$ F. Davoodi, "A Distributed Control Architecture For GOWON " California Institute of Technology, CIT File No.: CIT6392-P April 2012

${ }^{4}$ Burkhardt, M., Davoodi, F., Burdick, J.W., Davoudi, F., "Harvesting Analysis for Moball, A Self-Propelled Mobile Sensor Platform Capable of Long Duration Operation in Harsh Terrains," (to appear) Proc. IEEE Int. Conf. Robotics and Automation," May-June 2014, Hong Kong.

${ }^{5}$ J. Antol, P. Calhoun, J. Flick, G. Hajos, R. Kolacinski, D. Minton, R. Owens, J. Parker "Low Cost Mars Surface Exploration: The Mars Tumbleweed," NASA TM-2003-212411

${ }^{6}$ Goran Jurisa Basic, "Power-scavenging Tumbleweed Rover: Design, development, and testing of a Tumbleweed rover capable of generating electrical power by utilizing wind energy", A thesis submitted in conformity with the requirements for the degree of Master of Applied Science Graduate Department of Aerospace Engineering University of Toronto, 2010.

${ }^{7}$ K. Najafi, T. Galchev, E.E. Aktakka, R. L. Peterson, J. McCullagh, "Microsystems for Energy Harvesting," Invited Paper, 16th International Conference on Solid-State Sensors, Actuators, and Microsystems (IEEE Transducers), Beijing China, pp. 1845-1850, June 2011

${ }^{8}$ S. Dubowsky, K. Iagnemma, S. Liberatore, D.M. Lambeth, J.S. Plante, and P. J. Boston “A Concept Mission: Microbots for Large-Scale Planetary Surface and Subsurface Exploration", URL: http://www.geog.le.ac.uk/bgrg/lab.htm

${ }^{9}$ M. Pavone, J. Castillo, J. Hoff man, I. Nesnas," Spacecraft/Rover Hybrids for the Exploration of Small Solar System Bodie", URL: http://web.stanford.edu/ pavone/presentations/Pavone.NIAC2012.pdf

${ }^{10}$ Golombek, M., and Rapp, D., 1997, Size-frequency distributions of rocks on Mars and Earth analog sites: Implications for future landed missions: J. Geophys. Res., v. 102, p. 4117-4129.

${ }^{11}$ Committee on the Planetary Science Decadal Survey space Studies Board "Vision and Voyages for Planetary Science in the Decade 2013-2022", Division on Engineering and Physical Sciences. National Research Council of the National Academies

${ }^{12}$ Scanlon, Kathleen E., et al. "Volcano-ice interactions in the Arsia Mons tropical mountain glacier deposits." Icarus 237 (2014): 315-339. 